\title{
Study of Antibiotic Resistance among Uropathogens and Feacal Commensal Escherichia coli in Pediatrics Age Group to Revise the Role of Feacal Escherichia coli as a Reservoir of Antibiotic Resistance
}

\author{
Sujatha Karjigi* and R. Karthik \\ Department of Microbiology, Dr. B. R. Ambedkar Medical College and Hospital \\ Kadugondanahalli.Bangaluru-45Karnataka, India \\ *Corresponding author
}

\begin{abstract}
A B S T R A C T
Antibiotic resistance is a growing problem in pediatric urology as nonselective use of prophylaxis, and poor empiric prescribing practices. The feacal reservoir provides optimal

Keywords

Escherichia coli, Urinary tract infections, Antibiotic resistance, Pediatrics, Antibiotic prophylaxis, Antibiogram

Article Info

Accepted: 20 November 2018 Available Online: 10 December 2018 conditions for the transmission of resistance genes within and between bacterial species as gut is exposed to large number of bacteria. So, this study was undertaken for the knowledge of drug resistance among uropathogens and also revises the possible role of the intestinal microbiota as a reservoir of drug resistant bacteria. Out of 344 urine samples, from 0-14 age group 124 samples had significant growth yielded 134 isolates. Escherichia coli was most predominant isolate 71(53\%) Antibiotic resistance of Enterobacteriaceae isolates for cephalosporins was 48\%, Ampicillin 62\%, amoxiclav 50\%, Ciprofloxacin $19 \%$, levofloxacin $10.3 \%$, norfloxacin $43 \%$, Nitrofurantoin $6 \%$, gentamicin $12 \%$, amikacin $2.8 \%$, piperacillin -tazobactam $14 \%$ and carbapenems $2.8 \%$. Commensal E. coli from same patient showed $56.3 \%$ resistance to cephalosporin whereas urinary E. coli which was $53.5 \%$. Aminopenicillins resistance was also high in both isolates. Resistance to Norflox $21 \%$ in feacal E. coli and $53.5 \%$ in urinary E. coli, which was higher among other fluoroquinolones. Aminoglycoside, Nitrofurantoin, Carbapenems had 0-2\%antibiotic resistance. Resistance to some of the most commonly prescribed primary care antibiotics in feacal isolates suggest that one cause of carrying bacterial resistant feacal flora could be previous exposure to antibiotics. So Future research must prioritize understanding resistance in non-pathogenic bacteria, which could allow prescribing guidelines to be updated before it affects patient therapeutic outcomes.
\end{abstract}

\section{Introduction}

Urinary tract infection is common clinical problem occurring upto $10 \%$ female, $3 \%$ male infants and toddlers (Demuri et al., 2008). UTI accounts for majority of causes of unexplained fever in children below 3 years of age (Vigi et al., 2016). UTI with Significant number of pathogenic bacteria if not treated have greater risk of recurrent episodes and potentially serious consequences including cystitis, urethritis, pyelonephritis and renal scarring in children with vesico ureteric reflux (VUR). (Coulthard et al., 1997) Antibiotics are frequently prescribed everywhere in both empiric and regular therapy for UTI (Cullen $e t$ 
al., 2012). The etiologic agents of urinary tract infection are generally limited to the patient's own intestinal microbiota, with Escherichia coli, Enterococcus spp., KlebsiellaEnterobacter spp., and Proteus spp (Henry D Isenberg $2^{\text {nd }}$ ed 2007). The microbiota in the human gastrointestinal tract (GIT) is highly exposed to antibiotics, and may be an important reservoir of resistant strains and transferable resistance genes. Maternal GIT strains can be transmitted to the offspring, and resistances could be acquired from birth (de Vries et al., (2011). Under normal conditions, the gut "receives" a large number of bacteria from the hands, pharyngeal and nasal secretions, water, food, and beverages. Neonates acquire the environmental flora very quickly after birth (Guarino et al., 2012) and in a few cases develop sepsis after translocation of this new flora (Das et al., 2011).

Antibiotic resistance particularly important in low-income countries, where antibiotics are often available OTC, without the need for a prescription (Planta, 2007).

Misuse of antibiotics in this way can expose harmless or opportunistic bacteria to a plethora of antibiotics to which they develop resistance. The resistant organisms then contaminate the environment via the faces [86]. Cross transmission of the resistant strains can occur relatively easily if strong hygiene measures are not taken

Prior antibiotic therapy has been associated with subsequent infection by resistant organisms this pattern is consistent with UTI as well as other infection paper 1(14-19) and the pattern seems consistent irrespective of age, site of initial infection or whether the subsequent infection with the same or different organisms.

The empirical treatment is based on local antimicrobial resistance rates, illness severity.
The antibiotic sensitivity pattern of uropathogens in a population is essential to determine the empirical treatment, as use of inappropriate empirical treatment is found to be predictor of multidrug resistant uropathogens.

So this study was undertaken for the knowledge of drug resistance among uropathogens and also revises the possible role of the intestinal microbiota as a reservoir of drug resistant bacteria. Since $E$. coli was the predominant bacterial lineage found in the gut of young children, this bacterium was investigated for detailed drug resistance profile and was compared with antibiotic resistance of urinary $E$. coli isolates.

\section{Results and Discussion}

Out of 344 urine samples from 0-14 age group $124(36 \%)$ sample had significant growth.172 (50\%) no growth, $28(8.1 \%)$ insignificant bacteriuria20 (5.8\%) isolates had mixed growth of more than 3 types suggestive of improper sample collection (Table 1).

$124(36 \%)$ samples with significant growth yielded 134 isolates. Causative agents were $E$. coli (53\%), Klebsiella species (22.4\%), Proteus species (4.5\%) Enterobacter species (4.5\%), Pseudomonas spices (1.5\%), Staphylococcus aureus (1.5\%), Coagulase negative Staphylococcus aureus (CONS) (4.5\%), Enterococcus species (6.7\%) and Candida species (1.5\%) (Figure 1). Escherichia coli was most predominant isolate $71(53 \%)$ Antibiotic resistance of Enterobacteriaceae isolates for cephalosporins was 48\%, Ampicillin 62\%, amoxiclav 50\%, Ciprofloxacin 19\%, levofloxacin $10.3 \%$, norfloxacin $43 \%$, Nitrofurantoin $6 \%$, gentamicin $12 \%$, amikacin $2.8 \%$, piperacillin-tazobactam $14 \%$ and carbapenems $2.8 \%$ (Table 2). 
Seventy one Escherichia coli isolates from stool samples of patient who had significant bacteriuria were further processed for antibiotic resistance as it is most predominant bacteria (Table 3 ).

In our study gut commensal E. coli showed $56.3 \%$ resistance to cephalosporin whereas urinary E. coli which was $53.5 \%$. Aminopenicillins resistance was also high in both isolates. Resistance to Norfloxacin $21 \%$ in feacal $E$. coli and $53.5 \%$ in urinary $E$. coli, which was high among other fluoroquinolones. Aminoglycoside, Nitrofurantoin, Carbapenems had better antibiotic sensitivity.

In another study by Ashley Bryceet al antibiotic resistance of urinary pathogen was compared with $E$. coli from insignificant bacterial counts in urine which were considered as gut contaminants. In this study amoxicillin showed $49.37 \%$ resistance for urinary isolates and $37.2 \%$ for contaminant isolates but cephalosporin's resistance was only3.8\% compared to our study which was higher $53.3 \%$ in urinary isolates may be due to local prescribing patterns.

Study by Kousalya Prabahar et al showed Over $50 \%$ of antibiotic prescriptions were started on a clinical basis, without confirmation of a bacterial infection. 70\% prescriptions were for cephalosporins in pediatric in patients which supports our finding of increased cephalosporin resistant isolates.

Study by Karen et al., Characterize the E. coli fecal flora of UTI patients and healthy controls who had never had a UTI. E. coli colonies from each rectal swab were random amplified polymorphic DNA (RAPD) typed for clonality, dominance in the sample and correlation to the infecting UTI isolate in patients. The conclusion of this study was Feacal-UTI isolates from patients were more often associated with multidrug resistance compared to feacal-only clones, indicating a link between UTI virulence and antimicrobial resistance.

Table.1 Age, Gender and Culture Growth Distribution of urine samples

\begin{tabular}{|c|c|c|c|c|c|c|c|}
\hline \multirow[t]{2}{*}{$\begin{array}{l}\text { Age } \\
\text { group }\end{array}$} & \multirow[t]{2}{*}{ Growth } & \multirow{2}{*}{$\begin{array}{l}\text { Insignific } \\
\text { ant } \\
\text { growth }\end{array}$} & \multirow[t]{2}{*}{$\begin{array}{l}\text { No } \\
\text { growth }\end{array}$} & \multirow{2}{*}{\begin{tabular}{|l} 
mixed \\
growth \\
$>3$ \\
isolates
\end{tabular}} & \multirow[t]{2}{*}{ total } & \multicolumn{2}{|c|}{ SEX } \\
\hline & & & & & & Male & Female \\
\hline$<1$ year & $8(50 \%)$ & $2(12.5 \%)$ & $4(25 \%)$ & $2(12.5 \%)$ & $16(4.6 \%)$ & $5(31.3 \%)$ & $11(68.7 \%)$ \\
\hline 1-2years & $37(67.3 \%)$ & $2(3.6 \%)$ & $12(21.8 \%)$ & $4(7.2 \%)$ & $55(16 \%)$ & $18(32.7 \%)$ & $37(67.3 \%)$ \\
\hline $2-5 y r s$ & $33(32.4 \%)$ & $6(5.8 \%)$ & $60(58.8 \%)$ & $3(2.9 \%)$ & $102(30 \%)$ & $51(50 \%)$ & $41(40 \%)$ \\
\hline $5-10 \mathrm{yrs}$ & $30(27.5 \%)$ & $16(14.6 \%)$ & $56(51.4 \%)$ & $7(6.8 \%)$ & $109(32 \%)$ & $49(45 \%)$ & $60(55 \%)$ \\
\hline $10-14 y r s$ & $16(25.8 \%)$ & $2(3.2 \%)$ & $40(64.5 \%)$ & $4(6.4 \%)$ & $62(18 \%)$ & $34(54.8 \%)$ & $38(61.3 \%)$ \\
\hline Total & $124(36 \%)$ & $28(8.1 \%)$ & $172(50 \%)$ & $20(5.8 \%)$ & 344 & $157(45.6 \%)$ & $187(54.4 \%)$ \\
\hline
\end{tabular}


Table.2 Antibiotic resistance profile of urinary isolates

\begin{tabular}{|c|c|c|c|c|c|c|c|c|c|c|c|c|c|c|c|}
\hline E.COLI -71 & $40(56 \%)$ & $26(36.7 \%)$ & $38(53.5 \%)$ & $38(53.5 \%)$ & $38(53.5 \%)$ & $38(53.5 \%)$ & $14(19.7 \%)$ & $4(5.6 \%)$ & $38(53.5 \%)$ & $32(45 \%)$ & $2(2.8 \%)$ & $12(17 \%)$ & $2(2.8 \%)$ & $12(17 \%)$ & $2(2.8 \%)$ \\
\hline Klebsiella sp-30 & $30(100 \%)$ & $30(100 \%)$ & $14(46.6 \%)$ & $14(46.6 \%)$ & $14(46.6 \%)$ & $14(46.6 \%)$ & $4(13.3 \%)$ & $4(13.3 \%)$ & $8(26.6 \%)$ & $6(20 \%)$ & $2(6.6 \%)$ & $1(3.3 \%)$ & $\underline{\mathbf{0}}$ & $\underline{2(6.6 \%)}$ & $\underline{\mathbf{0}}$ \\
\hline Proteus spp-6 & 2(33.3\%) & $2(33.3 \%)$ & $4(66.6 \%)$ & $4(66.6 \%)$ & 4(66.6\%) & $4(66.6 \%)$ & $4(66.6 \%)$ & $4(66.6 \%)$ & $4(66.6 \%)$ & $2(33.3 \%)$ & $3(50 \%)$ & $2(33.3 \%)$ & $\underline{\mathbf{0}}$ & $2(33.3 \%)$ & $\underline{0}$ \\
\hline Total- 116 & 72(62\%) & $58(50 \%)$ & $56(48 \%)$ & $56(48 \%)$ & $56(48 \%)$ & $56(48 \%)$ & $\underline{22(19 \%)}$ & $12(10.3 \%)$ & $50(43 \%)$ & $40(34.4 \%)$ & $7(6 \%)$ & $15(12 \%)$ & $2(2.8 \%)$ & $16(14 \%)$ & $2(2.8 \%)$ \\
\hline
\end{tabular}

AMP-ampicillin,AMC-amoxyclav,CZ-cephazolin,CXM-Cefuroxime,CTR-ceftriaxone,CTX-Cefotaxime,CIP-Ciprofloxacin,LElevofloxacin,NX-Norfloxacin,COT-Cotrimoxazole,NIT-Nitrofurantoin,GEN-Gentamicin,AK-Amikacin,PIT-PiperacillinTazobactum,IMP-Imipenem,MRP-Meropenem,ERT-Ertapenem

Table.3 Antibiotic resistance of commensal feacal E. coli and uropathogen E. coli isolates

\begin{tabular}{|c|c|c|c|c|c|c|c|c|c|c|c|c|c|c|c|}
\hline 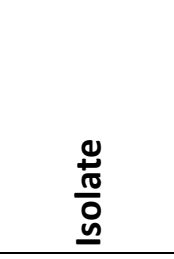 & 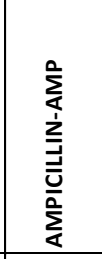 & 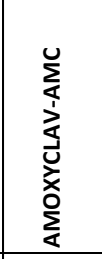 & 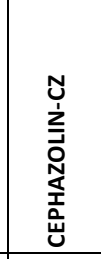 & 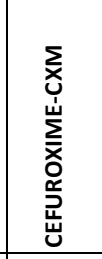 & 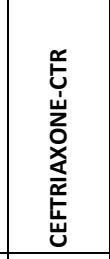 & 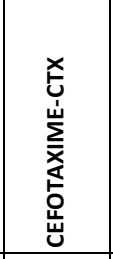 & 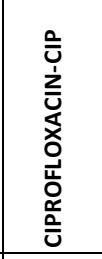 & 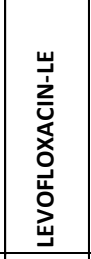 & 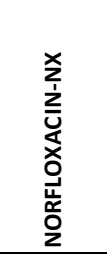 & 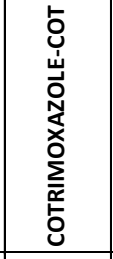 & 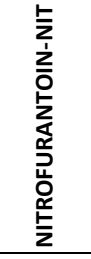 & 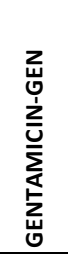 & 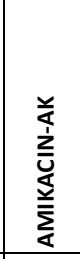 & 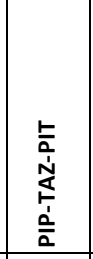 & 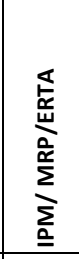 \\
\hline $\begin{array}{c}\text { Faecal E.COLI - } \\
71 \\
\end{array}$ & $45(63.4 \%)$ & \begin{tabular}{|c}
30 \\
$(42.3 \%)$ \\
\end{tabular} & $40(56.3 \%)$ & $40(56.3 \%)$ & $40(56.3 \%)$ & \begin{tabular}{|l|}
$40(56.3 \%)$ \\
\end{tabular} & $12(17 \%)$ & $6(8 \%)$ & $15(21 \%)$ & $29(40.8 \%)$ & 0 & $6(8 \%)$ & $5(7 \%)$ & $5(7 \%)$ & 0 \\
\hline $\begin{array}{c}\text { Urinary E.COLI - } \\
71\end{array}$ & $40(56 \%)$ & $26(36.7 \%)$ & $38(53.5 \%)$ & $38(53.5 \%)$ & $38(53.5 \%)$ & $38(53.5 \%)$ & $14(19.7 \%)$ & $4(5.6 \%)$ & $38(53.5 \%)$ & $32(45 \%)$ & $2(2.8 \%)$ & $\begin{array}{c}12 \\
(17 \%)\end{array}$ & $2(2.8 \%)$ & $12(17 \%)$ & $2(2.8 \%)$ \\
\hline
\end{tabular}

Figure.1 Causative agents of UTI(\%)

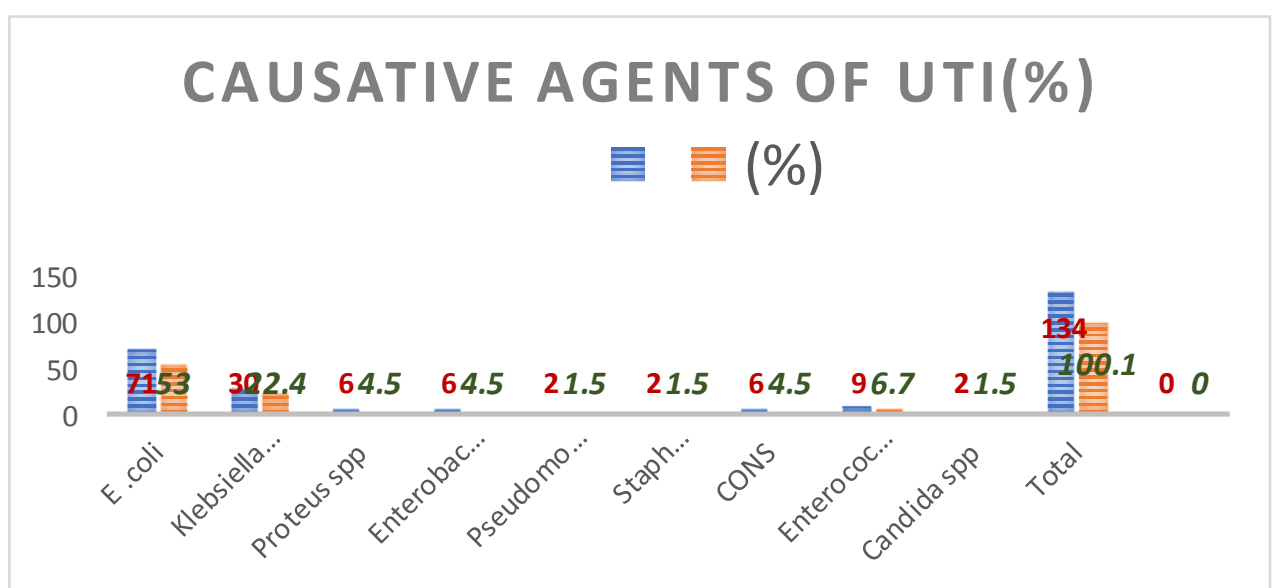

The present study isolated showed higher antibiotic resistance to Aminopenicillins, Norfloxacin, cotrimoxazole and Cephalosporin in both feacal and pathogenic
E. coli suggests poor empiric prescribing practices and nonselective use of prophylactic antibiotics this is particularly important in low-income countries, where antibiotics are 
often available over the counter, without the need for a prescription (Planta, 2007).

Misuse of antibiotics in this way can expose harmless or opportunistic bacteria to a plethora of antibiotics to which they develop resistance. Which contribute to or cause dysbiosis by directly eliminating the bacterial populations that confer colonization resistance to the intestinal microbiome and exerts selective pressure on both pathogenic and non-pathogenic bacteria which can alter the gut flora and subsequently the urinary tract. This can create an environment where resistant bacteria are able to thrive and persist, and may be an important reservoir of resistant strains and transferable resistance genes. Future research must prioritize understanding resistance in non-pathogenic bacteria, which could allow prescribing guidelines to be updated before it affected patient therapeutic outcomes.

\section{References}

Coulthard MG, et al., Occurrence of renal scars in children after their first referral for urinary tract infection. BMJ 1997; 315: 918-9.

Cullen IM et al., 2012. The changing pattern within 42033 E. coli isolates from nosocomial, community and urology specific UTI. https://www.ncbi.nlm.nih. gov/pubmed/21883861.) 3/6, $2 / 4$.

Das P et al., 2011 Colonization of the gut with Gram-negative bacilli, its association with neonatal sepsis and its clinical relevance in a developing country. J Med Microbiol 60:1651-1660.

De Vries LE, et al. (2011) The Gut as Reservoir of Antibiotic Resistance: Microbial Diversity of Tetracycline Resistance in Mother and Infant. PLoS ONE 6(6): e21644. doi:10.1371/journal.pone.002 1644)

DeMuri GP et al., 2008. Imaging and antimicrobial prophylaxis following the diagnosis of urinary tract infection in children. Pediatr Infect Dis J . 2008 Jun; 27(6): 553-4[PubMed ID: 18520594]

Guarino A et al., 2012, Wudy A, Basile F, Ruberto E, Buccigrossi V: Composition and roles of intestinal microbiota in children. J Matern Fetal Neonatal Med., 25(Suppl. 1): 63-66.

Henry d Isenberg clinical microbiology hand book $2^{\text {nd }}$ edition 20073.12/p374 Urine Cultures Kousalya Prabahar et al 2017 Asian Journal of Pharmaceutics, Jan-Mar 11 (1): $S 231$.

Karen L.et al., 2014, Faecal Escherichia coli from patients with $E$. coli urinary tract infection and healthy controls who have never had a urinary tract infection Journal of Medical Microbiology, 63, 582-589.

Planta MB 2007. The role of poverty in antimicrobial resistance. J Am Board Fam Med., 20(6): 533-9.

Vigi et al., 2016. High prevalence of multiple drug resistance among pediatric Escherichia coli infections. International Journal of Medical Research \& Health Sciences 5(10):166- 169.

\section{How to cite this article:}

Sujatha karjigi and Karthik, R. 2018. Study of Antibiotic Resistance among Uropathogens and Feacal Commensal Escherichia coli in Pediatrics Age Group to Revise the Role of Feacal Escherichia coli as a reservoir of Antibiotic Resistance. Int.J.Curr.Microbiol.App.Sci. 7(12): 2979-2983. doi: https://doi.org/10.20546/ijcmas.2018.712.341 